\title{
Frontières
}

\section{Ce qu'il est possible de défier}

\section{Ariane Brunet}

Volume 15, numéro 2, printemps 2003

Guerre, mort amère

URI : https://id.erudit.org/iderudit/1073821ar

DOI : https://doi.org/10.7202/1073821ar

Aller au sommaire du numéro

Éditeur(s)

Université du Québec à Montréal

ISSN

1180-3479 (imprimé)

1916-0976 (numérique)

Découvrir la revue

Citer ce document

Brunet, A. (2003). Ce qu'il est possible de défier. Frontières, 15(2), 54-55.

https://doi.org/10.7202/1073821ar d'utilisation que vous pouvez consulter en ligne.

https://apropos.erudit.org/fr/usagers/politique-dutilisation/ 


\section{Ce qu'il est possible de défier}

\section{Ariane Brunet, \\ coordonnatrice, programme Droits des femmes, Droit et Démocratie ${ }^{1}$.}

Il y a trois jours, je déambulais dans les rues de Kaboul péniblement consciente d'une solitude dont je m'accommode facilement en d'autres lieux. Là, cette singularité était lourde. J'étais seule dans mon genre dans ces rues où les femmes sont quasi absentes et où celles qui se risquent dans l'espace public le font, en grande majorité, de manière fantomatique. Ici, me suis-je dit, une femme ne peut être ellemême. Le défi en est un d'existence. Et si pour les femmes d'Afghanistan le défi est d'être, est-ce que je peux prétendre que d'autres femmes ailleurs défient un prétendu «nouvel» ordre fut-il politique ou militaire... surtout quand ce nouvel ordre construit sa légende, bâtit son mythe sur les ruines de l'Afghanistan?

Ce que je crois avoir compris à Kaboul, c'est que si nous ne pouvons prétendre défier le nouvel ordre du monde nous n'en sommes pas dupes.

La capacité d'endurance des femmes afghanes, leur confrontation brutale à la surenchère patriarcale, leur infinie créativité pour arriver à survivre témoignent de leurs ressources et de leur lucidité. Au cœur de chacune de mes rencontres avec elles, nous étions conscientes d'habiter en des mondes parallèles, une dimension dont leur regard invite à prendre toute la mesure.

L'Afghanistan, c'est en effet la rencontre d'un capitalisme global, d'une militarisation à son paroxysme, d'une reconstruction illusoire. L'engagement de la communauté internationale à aider (quand les États prétendent aider, l'idée est de donner afin de recevoir en retour le moment venu; soit, encorefaut-il que « l'aide » arrive...), aider à reconstruire la société afghane a pris après le 11 septembre 2001, une signification de taille.

Dans son rapport sur les droits humains et les efforts de reconstruction en Afghanistan publié en mai 2002, le Center for Economic and Social Rights écrit :

L'engagement public de la communauté

internationale d'aider les Afghans à reconstruire leur société revêt une signification mondiale, car il va créer un précédent pour établir la viabilité des promesses d'aide humanitaire après le 11 septembre. [...] Partout dans le monde, la reconstruction de l'Afghanistan est considérée comme un test révélateur en ce sens qu'elle permettra de constater si les valeurs universelles que sont les droits humains et le développement contribueront à définir les paramètres de la sécurité à l'échelle mondiale ou si ce sont les intérêts militaires étroitement définis par les États les plus puissants qui auront priorité (traduction de la revue) ${ }^{2}$.

Tout y est: assistance humanitaire, valeurs universelles, droits humains, développement, sécurité. Ce que le Center for Economic and Social Rights a omis, c'est que c'est aussi en Afghanistan que se joue l'utilisation de la carte féministe par les fondamentalistes chrétiens. La question des femmes fait aussi partie de l'arsenal de propagande militaire, comme l'a si bien démontré Katherine Viner dans son article Feminism as Imperialism, paru dans The Guardian le samedi 21 septembre 2002. Combien de gouvernements, en commençant par les États-Unis, ont soudainement dénoncé le sort réservé aux femmes afghanes afin de justifier leur intervention? Bush recycle le même discours pour sa prochaine intervention en Iraq! Il est là le défi.

Nous ne sommes pas dupes, mais il nous faudra nous élever contre l'utilisation du féminisme, des droits des femmes pour justifier les guerres à venir. Car, après, s'associer avec les femmes afghanes et s'entretenir sur les valeurs universelles, les droits humains prennent un tout autre sens. Oui, il nous faut assumer le caractère politique du féminisme. Force est de constater que Bush et sa femme l'auront, semble-t-il, compris avant nous.

La IV ${ }^{\mathrm{e}}$ rencontre ministérielle du Network sur la sécurité humaine a eu lieu cet été à Santiago du Chili. Les gouvernements ont voulu renouveler leur engagement à soutenir les efforts internationaux pour éradiquer le terrorisme «par une meilleure compréhension des racines de l'insécurité globale ». Dans le rapport de cette rencontre, pas le moindre mot sur la remilitarisation massive du globe... Il y a de quoi se poser de sérieuses questions sur ce nouvel appareillage linguistique. On y parle de l'importance de l'éducation sur les droits humains. Avait-on besoin de ce nouveau concept pour se rappeler des engagements de l'ONU à Vienne en 1993 lors de la Conférence mondiale sur les droits humains? Des dix ans d'une campagne d'éducation sur les droits?

S'il est stratégique de prendre diverses avenues dans le labyrinthe onusien quant à la promotion et à la défense des droits, se peut-il qu'il soit également opportun de se demander pourquoi la communauté internationale poursuit sa course folle de production d'instruments, de déclarations? Que signifie la fractionalisation de plus en plus sophistiquée des droits? Comment le discours féministe et surtout la pratique féministe y trouvent-ils leur compte?

La rencontre ministérielle de juillet 2002 propose de créer un index sur la sécurité humaine. On propose aussi de poursuivre la recherche sur les éléments constitutifs de la sécurité humaine. Par ailleurs, comme il se doit, en conclusion, on souligne l'adoption de la résolution 1325 du Conseil de sécurité sur les femmes, la paix et la sécurité et on souligne la contribution de cette résolution quant à l'application de mesures concrètes afin d'assurer aux femmes une participation pleine et entière à tous les aspects de la consolidation de la paix. Enfin, on se félicite d'une réunion sur l'intégration des droits des femmes dans le concept de sécurité humaine, réunion à Oslo en janvier 2001. Je sais, peu d'entre nous sommes au courant.

Combien de documents de cette espèce avons-nous lus depuis la Conférence mondiale sur les droits humains de 1993 ? Combien d'efforts pour assurer une approche sexo-spécifique? Combien de nouveaux documents nous permettant d'espérer une applicabilité même embryonnaire? Cette surenchère de concepts cacherait-elle une mauvaise volonté à respecter les principes de base, les droits fondamentaux? La dignité humaine n'implique-t-elle pas qu'on se comprenne quand on se parle?

L'idée n'est pas de discontinuer notre travail au sein des instances onusiennes, mais de mieux cibler nos interventions 
et surtout de questionner les nouvelles tendances, les nouveaux concepts qui superposent les droits les uns sur les autres, joute diplomatique qui permet trop souvent qu'un droit tombe dans l'oubli, que ce qui était évident hier devienne obscur aujourd'hui. Ce ne sont plus des familles de droits auxquelles nous avons à faire, mais à un labyrinthe où il nous faut nous enfoncer toute bougie éteinte.

Face à la construction de ce labyrinthe, bien peu d'intérêt pour la responsabilité des États, des corporations, du big business dans la militarisation de nos sociétés. Les tribunaux ad hoc du Rwanda et de l'exYougoslavie font leur petit bonhomme de chemin avec quelques ONG à leur trousse comme une tique sur un chien. Aujourd'hui, la Coalition sur les droits des femmes en situation de conflit, qui travaille depuis sept ans sur le Tribunal pénal international pour le Rwanda, est forcée de constater que la victoire d'Akayesu s'est révélée n'être qu'une simple note en bas de page de l'histoire. Nous n'avons pas réussi à faire naître un automatisme chez les procureurs afin que ceux-ci prennent en considération la violence sexuelle à chaque fois qu'il est approprié de le faire dans les cas présentés à la Cour. Et pourtant la justice, c'est un besoin fondamental, la cour pénale internationale est un des rares instruments qui, une fois mis en œuvre assurera transparence, équité, impartialité et une ébauche de réconciliation.

Tous ces questionnements, car nous nous devons de ne pas être dupes. Pour citer Arundhati et Roy dans un article intitulé Not Again paru dans The Guardian Weekly le 3-9 octobre 2002 :

À mesure que le fossé s'élargit entre les riches et les pauvres, le marché libre agit subrepticement et en coup de poing. Les sociétés multinationales constamment à l'affût de «bonnes occasions » susceptibles de rapporter d'énormes profits ne peuvent avoir leurs coudées franches dans les pays en voie de développement sans une connivence active de l'appareillage d'État. De nos jours, il faut que la mondialisation des sociétés puisse s'appuyer, dans les pays les plus pauvres, sur une confédération internationale de gouvernements loyaux, corrompus et préférablement autoritaires, pour faire adopter des réformes impopulaires et pour étouffer les mutineries. Il lui faut une presse qui se prétende libre et il lui faut aussi des tribunaux qui prétendront rendre la justice. Il lui faut des bombes nucléaires, des armées régulières, des lois plus sévères relativement à l'immigration et des garde-côtes plus vigilants pour veiller à ce que la mondialisation s'applique uniquement à l'argent, aux biens, aux brevets et aux services -

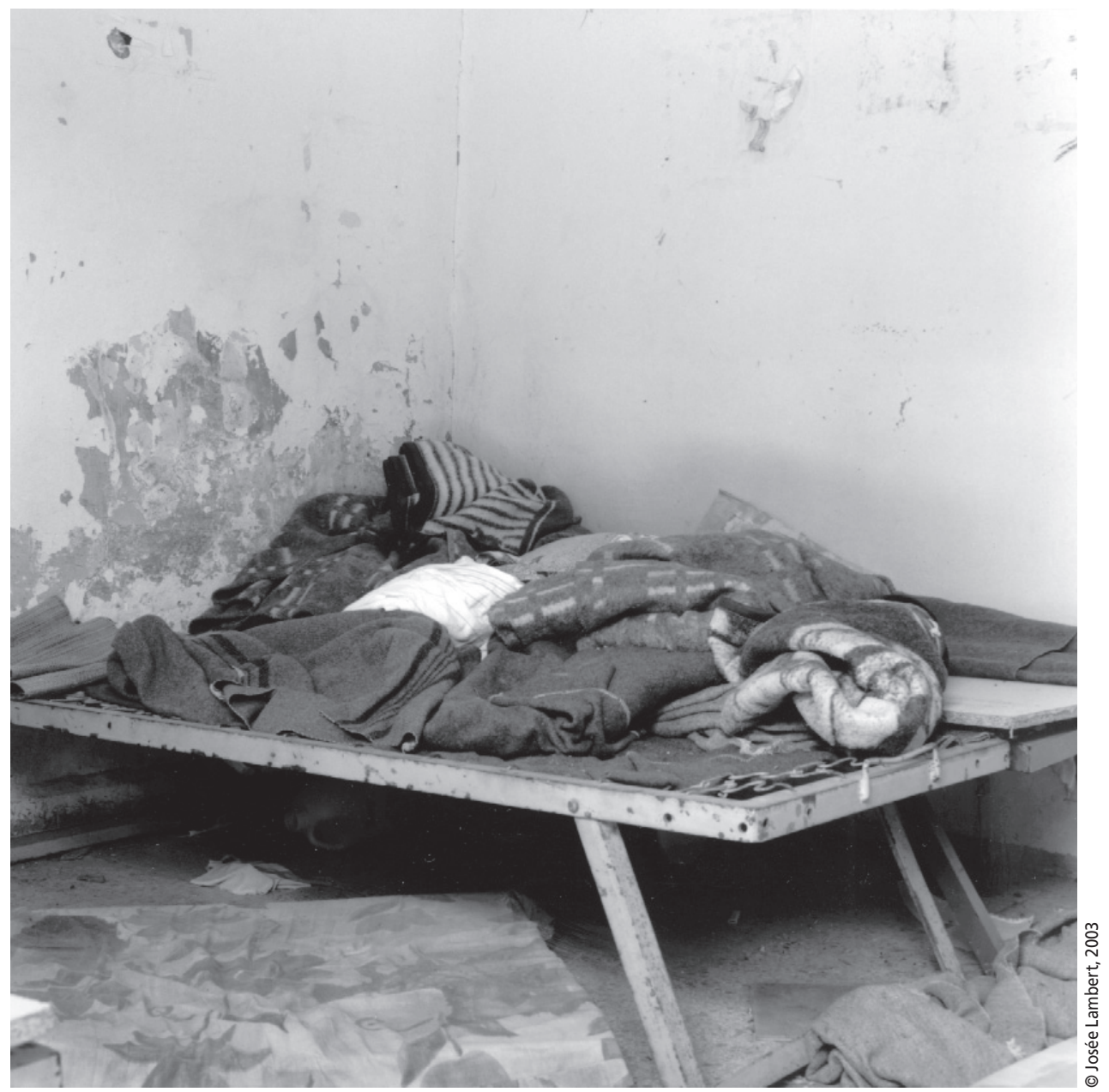

Dans les plis de vieilles couvertures sommeillent le précieux souvenir de la chaleur du corps d'un codétenu.

donc qu'on évite de mondialiser la libre circulation des personnes, le respect des droits humains, les traités internationaux contre la discrimination raciale, contre les armes chimiques et nucléaires, contre l'émission des gaz à effet de serre, contre les problèmes attribuables aux changements climatiques et, surtout, qu'on évite à tout prix une mondialisation de la justice. On se comporte comme si le moindre geste posé à l'échelle internationale en faveur de la simple obligation de rendre des comptes risquait de compromettre toute l'entreprise (traduction de la revue) $)^{3}$.

\section{Notes}

1. Cet article reprend une intervention prononcée dans le cadre du Forum AWID (Association for Woman's Rights in Development), à Guadalajara au Mexique lors de la plénière du 4 octobre 2002, intitulée: Les femmes mettent au défi le nouvel ordre politique et militaire (voir à ce sujet le site < http://fr. awid.org/ >) .
2. "The international community's public commitment to help Afghans rebuild their society has assumed global significance as a precedent for the viability of humanitarian engagement after September 11. [...] Throughout the world, the reconstruction of Afghanistan is seen as a litmus test for whether the universal values of human rights and development will help define the parameters of global security, or whether the narrow military interests of powerful states will predominate. »

3. "As the disparity between the rich and poor grows, the hidden fist of the free market has its work cut out. Multinational corporations on the prowl for "sweetheart deals" that yield enormous profits cannot push them through in developing countries without the active connivance of state machinery. Today, corporate globalisation needs an international confederation of loyal, corrupt, preferably authoritarian governments in poorer countries, to push through unpopular reforms and quell the mutinies. It needs a press that pretends to be free. It needs courts that pretend to dispense justice. It needs nuclear bombs, standing armies, sterner immigration laws, and watchful coastal patrols to make sure that it is only money, goods, patents and services that are globalised - not the free movement of people, not a respect for human rights, not international treaties on racial discrimination or chemical and nuclear weapons, or greenhouse gas emissions, climate change, or, God forbid, justice. It's as though even a gesture towards international accountability would wreck the whole enterprise.» 